01

\title{
Моделирование быстрой водородопроницаемости сплавов для мембранного газоразделения
}

\author{
() Ю.В. Заика, Н.И. Родченкова
}

Институт прикладных математических исследований Карельского научного центра РАН, 185910 Петрозаводск, Россия

e-mail: zaika@krc.karelia.ru

(Поступило в Редакцию 27 июля 2016 г.)

Методом измерения удельной водородопроницаемости исследуются различные сплавы, перспективные для использования в газоразделительных установках. Представлены нелинейная краевая задача водородопроницаемости в соответствии со спецификой эксперимента и ее модификации с учетом высокой скорости переноса. Обсуждено существенное отличие от квазиравновесной модели (приближение Ричардсона в предположении равновесного закона Сивертса в приповерхностном объеме). Модель апробирована на опубликованных экспериментальных данных по сплаву $\mathrm{Ta}_{77} \mathrm{Nb}_{23}$.

DOI: 10.21883/JTF.2017.05.44435.2014

\section{Введение}

Интерес к взаимодействию изотопов водорода с конструкционными материалами носит многоплановый характер [1-12]. Достаточно упомянуть задачи энергетики, защиты металлов от водородной коррозии, проектирования химических реакторов, ракетостроения. Гидриды позволяют удерживать большое количество водорода. С этим связаны перспективы использования водородных аккумуляторов без сверхвысоких давлений и низких температур. На обратимом легировании металлов водородом основаны пластифицирование и термоводородная обработка титановых сплавов. Некоторые частные задачи водородного материаловедения, близкие теме работы, исследованы в [13-15]. Энтузиасты говорят не только о водородной энергетике, но и о водородной экономике [7].

Для повышения эффективности экспериментальных исследований, решения прикладных задач и обобщений необходимы математические модели взаимодействия изотопов водорода с конструкционными материалами и методы их идентификации. Экспериментальный опыт показывает, что лимитирующими являются не только диффузионные процессы, но и физико-химические явления на поверхности $[1,2]$. Параметры переноса зависят и от технологических особенностей получения партии материала, поэтому вряд ли следует ориентироваться на получение „табличных данных“, нужны эффективные алгоритмы обработки экспериментальных кривых. В настоящей работе остановимся на методе проницаемости, учитывая лишь основные факторы и информативность эксперимента. Основой для проведенных исследований послужили работа [16] и данные по водородопроницаемости некоторых перспективных сплавов $[17,18]$.

\section{1. Математическая модель переноса}

\section{1. Динамические граничные условия}

Вначале кратко опишем эксперимент. Образец материала, нагретого до фиксированной температуры, явля- ется перегородкой вакуумной камеры. Предварительно проведена дегазация. В начальный момент времени на входной стороне скачкообразно создается давление молекулярного водорода. Измеряются падающее давление на входе и растущее давление водорода в выходной емкости. Информативность эксперимента ограничена, поэтому в модели водородопроницаемости учитываем только основные факторы для задачи фильтрации.

Рассмотрим перенос водорода сквозь образец (пластину толщины $l$ и площади $S$ ). Температура $T$ постоянна в течение эксперимента. Концентрация растворенного водорода (в атомарном состоянии) относительно мала, и диффузионный поток можно считать пропорциональным градиенту концентрации. Часть атомов Н взаимодействует с ловушками (микродефекты различной природы, включая микрополости), которые могут удерживать водород. Ориентируясь на прикладной смысл задачи и возможности метода проницаемости, ограничимся представлением об „ограниченном стоке“ без дополнительной детализации. В качестве модели диффузии с ограниченным захватом в объеме примем нелинейную систему уравнений

$$
\begin{aligned}
\frac{\partial c}{\partial t} & =D(T) \frac{\partial^{2} c}{\partial x^{2}}-f(T, z, c), \quad \frac{\partial z}{\partial t}=f(T, z, c), \\
f & \equiv a(T)\left[1-\frac{z(t, x)}{z_{\max }}\right] c(t, x)-a_{\text {out }}(T) z(t, x),
\end{aligned}
$$

где $c(t, x)$ - концентрация диффундирующего водорода (атомарного), $z(t, x)$ - концентрация захваченного диффузанта, $D-$ коэффициент диффузии, $a \equiv a_{\text {in }}$ и $a_{\text {out }}$ - коэффициенты поглощения и высвобождения атомов Н ловушками. Знак тождества часто будем использовать в смысле равенства по определению. Величину $z_{\max }$ считаем достаточно малой (рассматривается металлический сплав с высокой водородопроницаемостью), захват носит характер поправки и не требует более детального моделирования. В рабочем 
диапазоне температур $T \in[400,900] \mathrm{K}$ слагаемое $a_{\text {out }} z$ несушественно. В масштабе установления режима стационарной проницаемости относительно тонких мембран ловушки быстро насыщаются и не оказывают заметного влияния на проникающий поток. Поэтому, чтобы не „плодить“ второстепенные параметры модели, далее полагаем $a_{\text {out }}=0$. Этот коэффициент существен в экспериментах по полной дегазации при высоких температурах, и задание значения $a_{\text {out }}>0$ технически не усложняет численное решение краевой задачи. Величины $D, a$ зависят от температуры $T$ образца по закону Аррениуса с предэкспоненциальными множителями $D_{0}$, $a_{0}$ и энергиями активации $E_{D}, E_{a}(R-$ универсальная газовая постоянная):

$$
\begin{gathered}
D=D_{0} \exp \left\{-E_{D} /[R T(t)]\right\}, \\
a=a_{0} \exp \left\{-E_{a} /[R T(t)]\right\} .
\end{gathered}
$$

Начальные данные: в силу предварительной дегазации образца $c(0, x)=0, z(0, x)=0, x \in[0, l]$.

Из материального баланса потоков получаем следующие нелинейные граничные условия:

$$
\begin{aligned}
& -\frac{d Q_{\mathrm{in}}}{d t}=\left[\mu(T) s(T) p_{0}(t)-b(T) c_{0}^{2}(t)\right] S=-\left.S D \frac{\partial c}{\partial x}\right|_{x=0}, \\
& -\frac{d Q_{\mathrm{out}}}{d t}=\left[\mu(T) s(T) p_{l}(t)-b(T) c_{l}^{2}(t)\right] S=\left.S D \frac{\partial c}{\partial x}\right|_{x=l} .
\end{aligned}
$$

Здесь $Q_{\text {in }}(t), Q_{\text {out }}(t)$ - количества атомов водорода во входной емкости объема $V_{\text {in }}$ и выходной емкости объема $V_{\text {out }}, c_{0}(t) \equiv c(t, 0), c_{l}(t) \equiv c(t, l)$. Газообразный водород находится в молекулярной форме, но для единообразия, поскольку сквозь металлическую мембрану диффундирует атомарный водород, подсчет ведем в атомах. Согласно кинетической теории газов, плотность $J_{p}$ падающего на поверхность потока частиц, связана с давлением $p$ по формуле Герца-Кнудсена: $J_{p}=p / \sqrt{2 \pi m k T}$ $(k$ - постоянная Больцмана, $m$ - масса молекулы водорода). В масштабе рассматриваемых далее экспериментальных данных удобно в качестве единиц измерений выбрать $[l]=\mathrm{cm},[p]=$ Torr, откуда численно получаем зависимость $J_{p}=\mu p, \mu(T) \approx 2.474 \cdot 10^{22} / \sqrt{T}$ $\left([\mu]=1_{\mathrm{H}_{2}} /\left(\right.\right.$ Torr $\left.^{2} \mathrm{~s}\right),[T]=\mathrm{K}$, под знаком корня численное значение $T$ ). На поверхности происходят процессы физической адсорбции, хемосорбции, диссоциации молекул на атомы, растворения. Лишь малая часть „налетающих“ атомов Н окажется в абсорбированном состоянии в объеме мембраны. Это отражается множителем $s$. Таким образом, $\mu s p-$ результирующий поток атомов в объем сквозь поверхность без разделения на более элементарные стадии. Вместо $s$ можно написать $2 s$ и интерпретировать $s$ как долю абсорбируемых атомов $\mathrm{H}$.

Далее, $J_{0, l}=b c_{0, l}^{2}$ - это плотности потоков десорбции из образца (отклонение от квадратичности существенно лишь при экстремальных температурах), $b$ - коэффициент десорбции, $[b]=\mathrm{cm}^{4} / \mathrm{s}$. По контексту слово „плотность“ часто будем опускать. Для $s$ и $b$ также предполагаем аррениусовскую зависимость от температуры. По крайней мере формально, в экспоненте „энергия активации“ $E_{s}$ может оказаться и отрицательной величиной как линейная комбинация энергий активаций и теплот поверхностных процессов на пути „из газа в раствор“. Если с обеих сторон мембраны поддерживать постоянное давление насыщения $\bar{p}$ молекулярного водорода при постоянной температуре $T$, то в итоге установится равновесная концентрация $\bar{c}$ растворенного атомарного диффузионно подвижного водорода. Из модели (2), (3), приравнивая производные к нулю, получаем $\bar{c} \propto \sqrt{\bar{p}}: \bar{c}=\Gamma \sqrt{\bar{p}}, \Gamma \equiv \sqrt{\mu s / b}$.

Уточним экспериментальные условия. Объемы $V_{\text {in,out }}-$ несколько литров, толщина мембраны $l$ меньше $1 \mathrm{~mm}$, площадь $S-$ около $1 \mathrm{~cm}^{2}$, давление напуска $p_{0}(0)$ - несколько десятков Torr. Диапазон $\left[p_{\min }, p_{\max }\right]$ невелик, ограничимся $z_{\max }=\sigma \bar{c}, \sigma \leq 0.1$. Это не приведет к нарушению закона Сивертса $(\bar{c}+$ $\left.+z_{\max } \propto \sqrt{\bar{p}}\right)$, причем $\bar{c}+z_{\max } \approx \bar{c}=\Gamma \sqrt{\bar{p}}$ в пределах экспериментальной точности. Остается определить величины $Q_{\text {in }}, Q_{\text {out }}$. В масштабе времени переноса газ находится в термодинамическом квазиравновесии с поверхностью, поэтому воспользуемся формулой $N=$ $=p V /(k T)$. Здесь $N-$ количество частиц газа, занимаемого объем $V$ при температуре $T_{V}$ и давлении $p$ (в системе СИ $[p]=\mathrm{Pa},[V]=\mathrm{m}^{3},[k]=\mathrm{J} / \mathrm{K}$ ). С учетом соотношений Torr $=133.322 \mathrm{~Pa}, \mathrm{~Pa}=\mathrm{J} / \mathrm{m}^{3}$ (формально) получаем для соответствующих давлений и объемов в граничных условиях (2), (3) $Q=2 N=\alpha p V / T, \alpha \approx$ $\approx 1.931 \cdot 10^{19}$. Здесь $p, V, T$ означают численные значения в выбранных единицах (Torr, $\mathrm{cm}^{3}, \mathrm{~K}$ ).

\section{2. Функция стока и возможные модификации}

Остановимся подробнее на функции стока $f(T, z, c)=$ $=a\left[1-z z_{\max }^{-1}\right] c \quad([a]=1 / \mathrm{s})$. Скорость поглощения атомов Н увеличивается с ростом концентрации диффузанта и уменьшается по мере заполнения ловушек. В принципе величину $z_{\max }$ в физически разумном диапазоне можно считать независимым параметром модели. Пропорция $z_{\max }=\sigma \bar{c}$ принята с учетом того, что материал для мембранного газоразделения достаточно однороден и захват носит характер малой поправки. Общая растворимость определяется концентрацией $\bar{c}+z_{\max } \propto \bar{p}$. Это согласуется с известным мнением [19, с. 512]: „. . .часть водорода прочно удерживается дефектами решетки и поэтому не должна учитываться при определении концентрации, градиент которой входит в уравнение закона Фика, т. е. часть водорода является полуинертной примесью. Закон Фика применим только к оставшейся части“. Итак, эффект захвата учтен без дополнительных параметров в соответствии с ограниченной информативностью метода проницаемости. Другое дело, например, пористый вольфрам [3], когда микропоры достаточно велики для рекомбинации атомов водорода в молекулы. Это уже требует по существу более детального моделирования захвата. 
При необходимости можно учесть емкость поверхности. Кроме того, могут образовываться заметные оксидная пленка и гидридная фаза (по существу слой другого материала), что существенно сказывается на водородопроницаемости. В принципе можно считать мембрану трехслойной и учесть динамическое накопление в приповерхностных слоях, в том числе и за счет гидридообразования. Появятся дополнительные коэффициент диффузии и коэффициенты в условиях сопряжения на стыках слоев (равенство диффузионных потоков, но скачки концентраций)... Далее, при большом перепаде давлений мембрана испытывает изгиб (на входе сжатие, на выходе растяжение). Простейший вариант (без учета напряжений и деформаций) - считать значения $s, b$ независимыми и различными при $x=0, l$. При такой детализации образуется „снежный ком“ параметров с неизвестными a priori значениями. Поскольку задача связана с совершенствованием технологий газоразделения, основной смысл имеет оценка „интегральных“ показателей, фиксируемых в экспериментах проницаемости. Кроме того, водород является чрезвычайно подвижной фазой внедрения и измерения с высокой точностью затруднительны. В этом контексте остановимся на принятой модели, учитывающей лишь лимитирующие факторы.

\section{3. Безразмерная краевая задача}

Приведем модель в компактной форме

$$
\begin{gathered}
\frac{\partial c}{\partial t}=D(T) \frac{\partial^{2} c}{\partial x^{2}}-a(T)\left[1-\frac{z(t, x)}{z_{\max }}\right] c(t, x), \\
\frac{\partial z}{\partial t}=a(T)\left[1-\frac{z(t, x)}{z_{\max }}\right] c(t, x), \\
c(0, x)=0, \quad z(0, x)=0, \\
\mu(T) s(T) p_{0, l}(t)-b(T) c_{0, l}^{2}(t)=\left.\mp D(T) \frac{\partial c}{\partial x}\right|_{x=0, l}, \\
\frac{d Q_{\text {in,out }}}{d t}=-\left[\mu(T) s(T) p_{0, l}(t)-b(T) c_{0, l}^{2}(t)\right] S, \\
Q_{\text {in,out }}=\alpha p_{0, l}(t) V_{\text {in,out }} T^{-1} .
\end{gathered}
$$

Выберем нормировки. Температура $T$ фиксирована. По максимальному давлению $\bar{p}_{0}=p_{0}(0)$ определим соответствующие равновесную концентрацию диффундирующего водорода $\bar{c}=\Gamma \sqrt{\bar{p}_{0}}$ и количества атомов $\bar{Q}_{\text {in }}=\alpha \bar{p}_{0} V_{\text {in }} / T, \bar{Q}_{\text {out }}=\alpha \bar{p}_{0} V_{\text {out }} / T$. Перейдем к безразмерным координате $y=x / l$, концентрациям $u=c / \bar{c}$, $v=z / \bar{c} \quad\left(z_{\max }=\sigma \bar{c} \ll \bar{c}\right) \quad$ и времени $\tau: \quad t=D^{-1} l^{2} \tau$ $\left([D]=\mathrm{cm}^{2} / \mathrm{s}\right)$. Получаем следующую краевую задачу:

$$
\begin{gathered}
\frac{\partial u}{\partial \tau}=\frac{\partial^{2} u}{\partial y^{2}}-\tilde{a}\left[1-\frac{v}{v_{\max }}\right] u, \\
\frac{\partial v}{\partial \tau}=\tilde{a}\left[1-\frac{v}{v_{\max }}\right] u,
\end{gathered}
$$

$$
\begin{gathered}
u, v \in[0,1], \quad y \in(0,1), \\
u(0, y)=0, \quad v(0, y)=0, \quad y \in[0,1], \\
\tilde{a}(T) \equiv a l^{2} D^{-1},\left.\quad u_{0,1}(\tau) \equiv u(\tau, y)\right|_{y=0,1}, \\
\tilde{p}_{0,1}(\tau) \equiv p_{0, l}(t) \bar{p}_{0}^{-1}, \\
W(T) \equiv b \bar{c} l D^{-1}=\bar{P}_{0} l[D \bar{c}]^{-1}, \quad \bar{P}_{0} \equiv \mu s \bar{p}_{0}, \\
\left.\tilde{p}_{0}-u_{0}^{2}\right]=-\left.\frac{\partial u}{\partial y}\right|_{y=0}, \quad W\left[\tilde{p}_{1}-u_{1}^{2}\right]=\left.\frac{\partial u}{\partial y}\right|_{y=1}, \\
\bar{Q}_{\text {in }} \frac{d \tilde{p}_{0}}{d \tau}=-\bar{Q}_{m} W\left[\tilde{p}_{0}-u_{0}^{2}\right], \quad \tilde{p}_{0}(0)=1, \\
\bar{Q}_{\text {in }} \equiv \alpha V_{\text {in }} \bar{p}_{0} T^{-1}, \\
\bar{Q}_{\text {out }} \frac{d \tilde{p}_{1}}{d \tau}=-\bar{Q}_{m} W\left[\tilde{p}_{1}-u_{1}^{2}\right], \quad \tilde{p}_{1}(0)=0, \\
\bar{Q}_{\text {out }} \equiv \alpha V_{\text {out }} \bar{p}_{0} T^{-1} .
\end{gathered}
$$

Величина $\bar{Q}_{m}$ равна количеству атомов водорода (диффузионно подвижного) в образце в режиме равновесного насыщения при давлении $\bar{p}_{0}=p_{0}(0)$ и температуре $T$. Отметим, что транспортный параметр $W$ [3] играет определяющую роль при анализе варианта метода проницаемости, когда на выходе производится постоянное вакуумирование (метод прорыва).

Замечание 1. Формально нулевые начальные и граничное условие баланса $W\left[\tilde{p}_{0}-u_{0}^{2}\right]=-\left.\partial_{y} u\right|_{y=0}$ не согласованы при $t \rightarrow+0\left(\tilde{p}_{0}(0)=1, u_{0}(0)=0, \partial_{y} u(0,0)=0\right)$. На самом деле „мгновенный“ напуск водорода на входе длится некоторое время, пусть и пренебрежимо малое. В алгоритме решения краевой задачи, изложение которого мы здесь опускаем, это фактически учтено. Математически, безотносительно к вычислительному алгоритму, следует вести речь в терминах теории обобщенных решений.

\section{2. Результаты численного моделирования}

Входные величины: $T=400^{\circ} \mathrm{C}, \quad l=0.014 \mathrm{~cm}, \quad S=$ $=0.785 \mathrm{~cm}^{2}, V_{\text {in }}=3000 \mathrm{~cm}^{3}, V_{\text {out }}=1750 \mathrm{~cm}^{3}$. Результат аппроксимации экспериментальных данных по сплаву $\mathrm{Ta}_{77} \mathrm{Nb}_{23}$ [16] (рис. 1) модельными кривыми (входного и выходного давлений молекулярного водорода) представлен на рис. 1. Прокомментируем рис. 2. Согласно принятой модели равновесная концентрация $\bar{c}$ при условиях насыщения $p=$ const, $T=$ const определяется (после приравнивая к нулю производных) как $\bar{c}=\Gamma \sqrt{p}$, где $\Gamma \equiv \sqrt{\mu s / b}-$ коэффициент растворимости. Но следует помнить, что учтен только диффузионно подвижный растворенный атомарный водород. Полная дегазация определяется концентрацией $\bar{c}+z_{\max }=\Gamma_{\max } \sqrt{p}$. В принятой модели $\Gamma_{\max }=\Gamma[1+\sigma]$ при сохранении закона Сивертса $\bar{c}+z_{\max } \propto \sqrt{p}$. По сравнению с рис. 1 на 


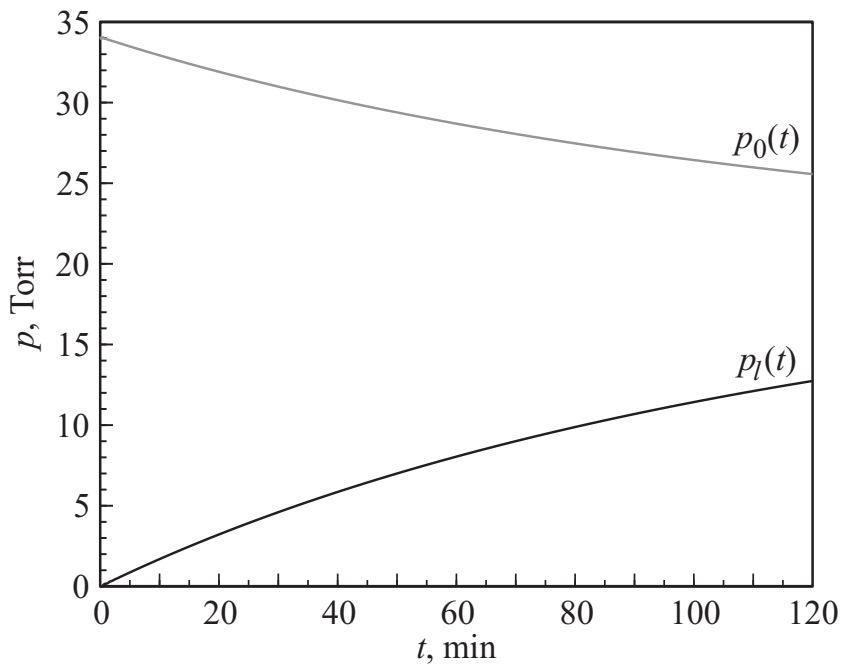

Рис. 1. Аппроксимация давлений, $T=400^{\circ}$ C. $D=5.5 \cdot 10^{-5}$, $b=2.1 \cdot 10^{-22}, \quad s=5.7 \cdot 10^{-5}, \quad \mu=9.55 \cdot 10^{20}, \quad a=0.1$, $\sigma=0.1, z_{\max }=0.1 \bar{c}=9.4 \cdot 10^{18}, \Gamma=\sqrt{\mu s / b}=1.6 \cdot 10^{19}, \Phi=$ $=D \Gamma=8.8 \cdot 10^{14}, D \Gamma_{\max }=9.7 \cdot 10^{14}$.

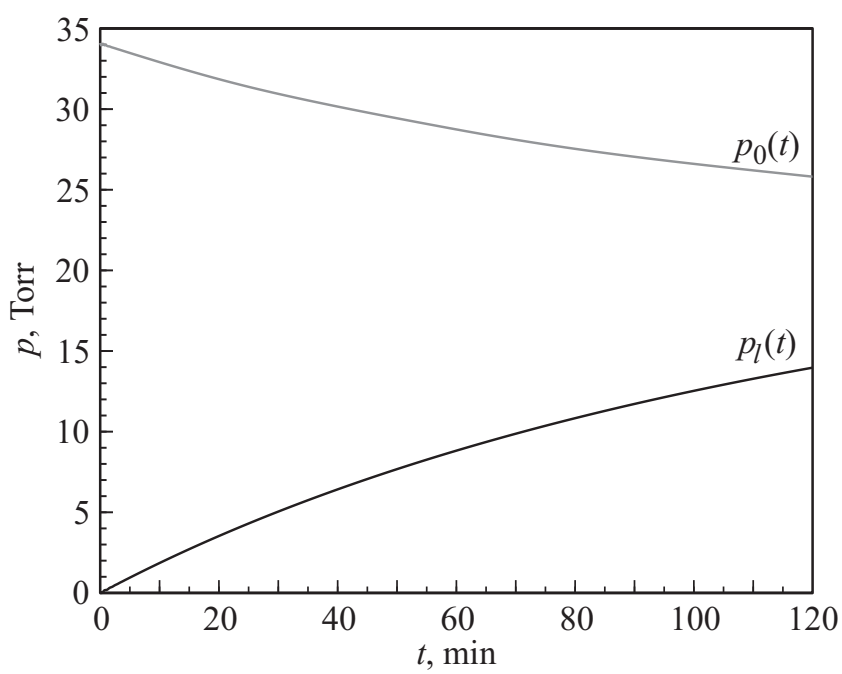

Рис. 2. Давления в случае $z_{\max }=10 \bar{c} . \quad T=400^{\circ} \mathrm{C}$, $D=5.5 \cdot 10^{-5}, \quad b=2.1 \cdot 10^{-22}, \quad s=5.7 \cdot 10^{-5}, \quad a=0.1$, $\sigma=10, z_{\max }=10 \bar{c}=9.4 \cdot 10^{20}, \Gamma=\sqrt{\mu s / b}=1.6 \cdot 10^{19}, \Gamma_{\max }=$ $=\Gamma(1+\sigma)=1.76 \cdot 10^{20}, \Phi=D \Gamma=8.8 \cdot 10^{14}, D \Gamma_{\max }=9.7 \cdot 10^{15}$.

рис. 2 с гипотетическим значением $z_{\max }=10 \bar{c}$ фиксирована общая растворимость на порядок больше. Если регистрировать установившийся поток водородопроницаемости $J=-D \partial_{x} c$ в эксперименте прорыва, когда $p_{0}(t)=p=\mathrm{const}$, а на выходе вакуумирование, то в предположениях $c_{0}=\bar{c}_{0}=\Gamma \sqrt{p}, c_{l}=0$ имеем $J=\Phi \sqrt{p} / l, \Phi \equiv D \Gamma=D \sqrt{\mu s / b}$. Если в серии экспериментов $(T=$ const, варьируется $p)$ с приемлемой точностью регистрируется пропорциональность $J \propto \sqrt{p}$, то по значениям $p, J$ определяют так называемый коэффициент водородопроницаемости $\Phi$ (исходя из формулы $J=\Phi \sqrt{p} / l$ безотносительно к принятой модели и информации о значениях $D, \Gamma)$. Проницаемость определяется диффундирующим водородом (в модели $\Phi=D \Gamma)$. Если же проницаемость $\Phi$ вычислять по имеющимся независимым значениям коэффициентов диффузии и растворимости (эксперименты различны и растворимость общая, с учетом захваченного водорода), то можем получить $D \Gamma_{\max } \gg D \Gamma$. По-видимому, это одна из причин разброса данных по растворимости и проницаемости. Проблема в том, что на тонких мембранах в режиме проницаемости трудно обнаружить „пропажу“ водорода в ловушках даже при большой их емкости, существенно влияющей на общую растворимость в материале (при пересчете на $\mathrm{cm}^{3}$ и $\mathrm{m}^{3}$ в условиях $\left.l, S \ll 1\right)$.

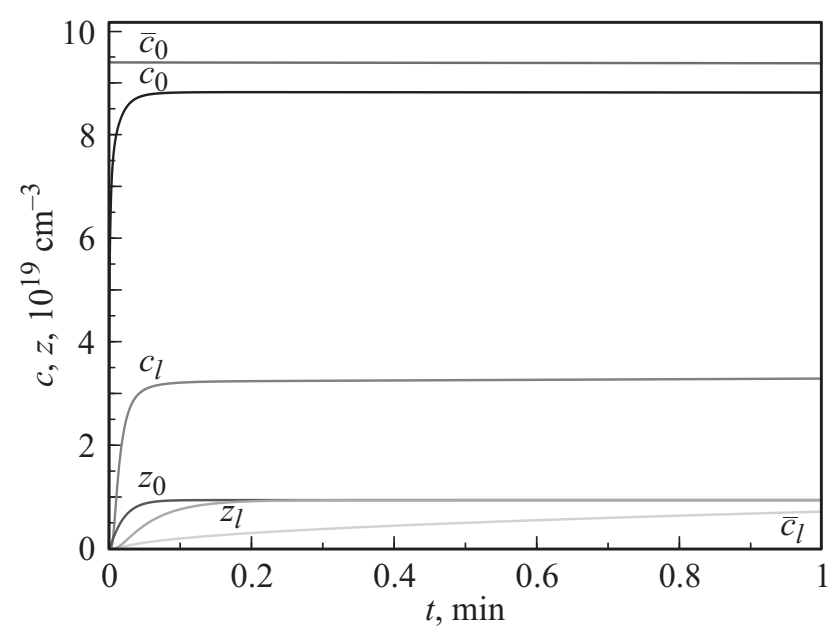

Рис. 3. Приповерхностные концентрации, $1 \mathrm{~min}$. $D=5.5 \cdot 10^{-5}, \quad b=2.1 \cdot 10^{-22}, \quad s=5.7 \cdot 10^{-5}, \quad \bar{p}_{0}=34.1$, $\mu=9.54 \cdot 10^{20}, \quad a=0.1, \quad \sigma=0.1, \quad z_{\max }=0.1 \bar{c}=9.4 \cdot 10^{18}$, $\Gamma=\sqrt{\mu s / b}=1.6 \cdot 10^{19}, \Gamma_{\max }=\Gamma(1+0.1)=1.77 \cdot 10^{19}$.

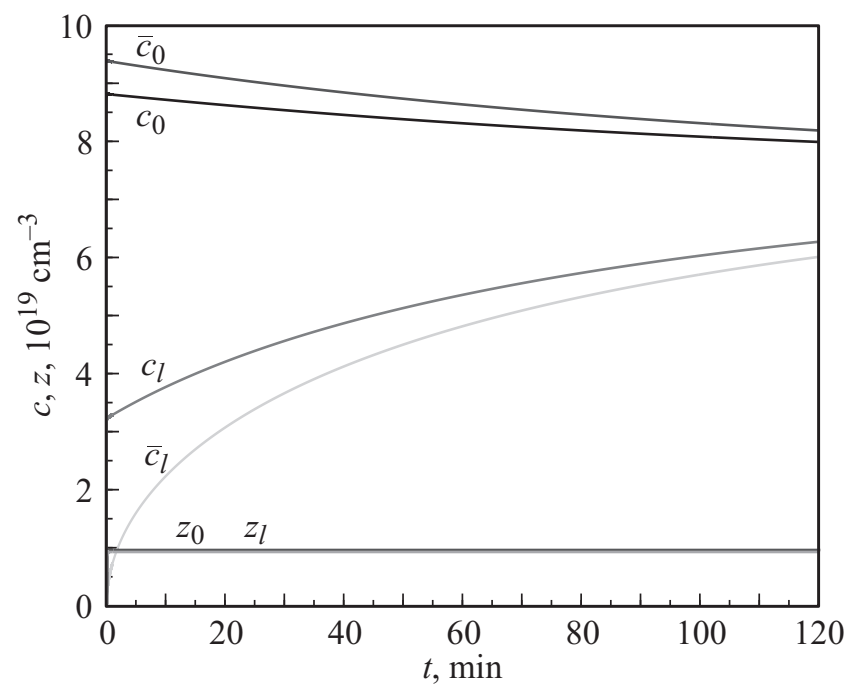

Рис. 4. Приповерхностные концентрации, $120 \mathrm{~min}$. $D=5.5 \cdot 10^{-5}, \quad b=2.1 \cdot 10^{-22}, \quad s=5.7 \cdot 10^{-5}, \quad \bar{p}_{0}=34.1$, $\mu=9.54 \cdot 10^{20}, \quad a=0.1, \quad \sigma=0.1, \quad z_{\max }=0.1 \bar{c}=9.4 \cdot 10^{18}$, $\Gamma=\sqrt{\mu s / b}=1.6 \cdot 10^{19}, \Gamma_{\max }=\Gamma(1+0.1)=1.77 \cdot 10^{19}$. 


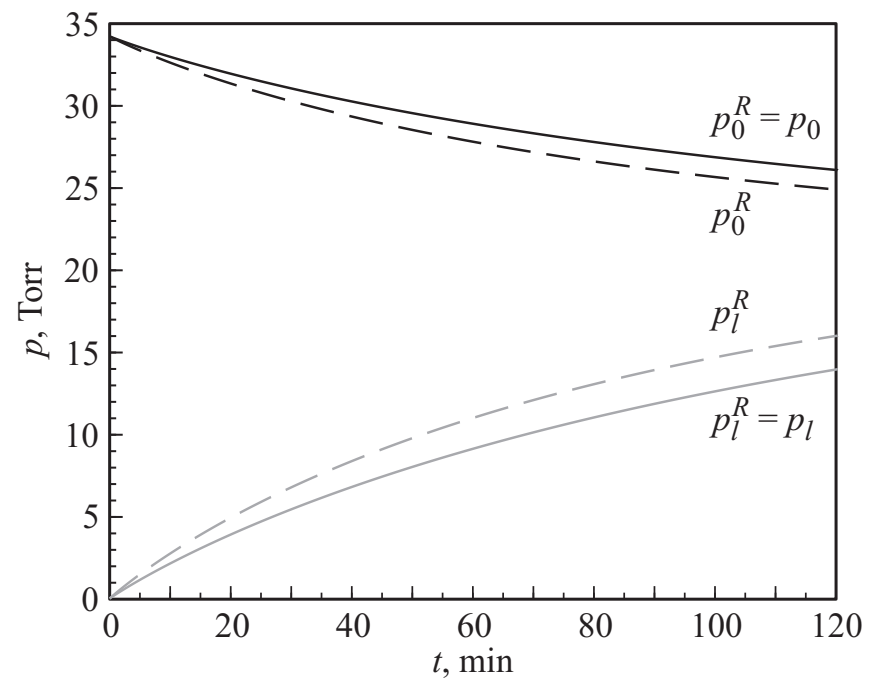

Рис. 5. Квазиравновесная модель, $T=400^{\circ}$ C. $D=5.5 \cdot 10^{-5}$, $b=2.1 \cdot 10^{-22}, s=5.7 \cdot 10^{-5}$, штриховые кривые $-\Gamma=$ $=\sqrt{\mu s / b}=1.6 \cdot 10^{19}, \Phi=D \Gamma=8.8 \cdot 10^{14}$, сплошные кривые $-\Gamma=1.2 \cdot 10^{19}, \Phi=6.6 \cdot 10^{14}$.

Перейдем к анализу динамики объемных концентраций в рассматриваемом эксперименте „сообщающихся сосудов“. Начнем с приповерхностных концентраций $(x=0, l)$ (рис. 3,4$)$. В течение минуты происходит заполнение ловушек: $z_{0} \approx z_{l} \approx z_{\max }$. Концентрации $c_{0}(t)=c(t, 0), c_{l}(t)=c(t, l)$ быстро стабилизируются, но, как видно в масштабе двух часов, это локально: происходит смена переходного режима всплеска на медленный тренд. Сравнимое время стабилизации $c_{l}$ (по отношению к $c_{0}$ ) объясняется быстрой водородопроницаемостью сплава и $l \ll 1$. Высокая скорость переходных процессов ожидаема, поскольку характеристическое время диффузии $l^{2} / D\left(D \sim 10^{-5}\right)$ в пределах $10 \mathrm{~s}$. Общая концентрация определяется суммой $c+z$. Квазиравновесные (сивертсовские) концентрации диффундирующего водорода $\bar{c}_{0, l} \propto \sqrt{p_{0, l}}$ определяются по давлениям молекулярного водорода соотношениями $\mu s p_{0, l}=b c_{0, l}^{2}: \bar{c}_{0, l}(t)=\Gamma \sqrt{p_{0, l}(t)}$. С учетом захвата следует оперировать суммами $\bar{c}_{0, l}(t)+z_{\max }$ и $c_{0, l}(t)+z_{0, l}(t)$. По рис. 4 можно оценить, насколько рассогласование концентраций $c_{l}-\bar{c}_{l}$ на выходе существенно больше входного $\bar{c}_{0}-c_{0}$. Лишь асимптотически (по мере приближения к равновесию) происходит сближение этих величин. В контексте задачи газоразделения нас в первую очередь интересует проникающий поток. Но аппроксимация градиента $\partial_{x} c$ разностным отношением $\left[c_{l}(t)-c_{0}(t)\right] / l$ ухудшается переходом к квазиравновесным оценкам как на входе $\left(\bar{c}_{0}>c_{0}\right)$, так и на выходе $\left(\bar{c}_{l}<c_{l}\right)$.

Проиллюстрируем текущие рассуждения. Примем базовую модель за „начало отсчета“(разд. 1.3) и упростим ее в предположении квазиравновесности приповерхностных концентраций и линейного распределения в объеме (приближение Ричардсона для проникающего потока):

$$
\begin{aligned}
\frac{d Q_{\text {in,out }}}{d t} & = \pm\left. S D(T) \frac{\partial c}{\partial x}\right|_{x=0, l}= \pm S D \frac{c_{l}(t)-c_{0}(t)}{l} \\
& = \pm S D \Gamma \frac{\sqrt{p_{l}(t)}-\sqrt{p_{0}(t)}}{l} \\
Q_{\text {in,out }}= & \alpha p_{0, l} V_{\text {in,out }} T^{-1} \Rightarrow \frac{d p_{0, l}}{d t} \\
= & \pm S D \Gamma\left[\alpha V_{\text {in,out }} l\right]^{-1} T\left[\sqrt{p_{l}(t)}-\sqrt{p_{0}(t)}\right] .
\end{aligned}
$$

Решая численно систему дифференциальных уравнений для $p_{0, l}(t)$ с начальными данными $p_{0}(0)=\bar{p}_{0}, p_{l}(0)=0$, получаем аппроксимацию (штриховые линии на рис. 5) экспериментальных давлений. Достаточно интегрировать одно уравнение в силу

$$
\begin{aligned}
\dot{p}_{l}(t) & =-V_{\text {in }} V_{\text {out }}^{-1} \dot{p}_{0}(t) \Rightarrow p_{l}(t) \\
& =V_{\text {in }} V_{\text {out }}^{-1}\left[\bar{p}_{0}-p_{0}(t)\right] .
\end{aligned}
$$

Результат неудовлетворителен, если сравнить с аппроксимацией базовой моделью (рис. 1 и рис. из [16]). Но ценою существенной вариации „истинных“ значений параметров можно добиться хорошего приближения (сплошные модельные линии на рис. 5). Коэффициент проницаемости (данные приведены на рис. 5) равен $\Phi=D \Gamma \approx 8.812 \cdot 10^{14}$. При подгонке по квазиравновесной модели получается $6.639 \cdot 10^{14}$. Сравнивая с отношением $\left[\bar{c}_{0}-\bar{c}_{l}\right] /\left[c_{0}-c_{l}\right] \approx 1.3$ (рис. 4 ), заключаем, что значительно более точной была бы квазистационарная модель $\dot{p}_{0, l}(t)= \pm S D\left[\alpha V_{\text {in,out }} l\right]^{-1} T\left[c_{l}(t)-c_{0}(t)\right]$. Но информации о граничных концентрациях нет, и вынужденная „сивертсовская“ подстановка $c_{0, l}=\Gamma \sqrt{p_{0, l}}$ занижает коэффициент проницаемости при аппроксимации давлений.

Замечание 2. В квазиравновесную модель входит только комплекс параметров переноса $\Phi=D \Gamma=D \sqrt{\mu s / b}$. При вариациях значений $D, b, s$, сохраняющих коэффициент проницаемости $\Phi$, модельные графики давлений не изменятся. Для тонких мембран с большой водородопроницаемостью ситуация практически квазистационарна (линейные распределения концентрации), но отлична от квазиравновесной (из-за $c_{0, l} \neq \bar{c}_{0, l}$ ). С ростом времени квазистационары все ближе к квазиравновесным распределениям. Это означает, что обратная задача параметрической идентификации плохообусловлена (в смысле слабой чувствительности модельных давлений к вариациям параметров, сохраняющих значение $\Phi)$.

Подчеркнем, что под квазистационаром мы понимаем практически линейный по $x$ профиль концентрации $c(t, x)$ с насыщенными ловушками, который относительно медленно меняется со временем. Квазиравновесным считаем такой квазистационар, который характеризуется заменой $c_{0, l}(t)$ на „сивертсовские“ концентрации $\bar{c}_{0, l}=\Gamma \sqrt{p_{0, l}(t)}\left(z=z_{\max }\right)$. 


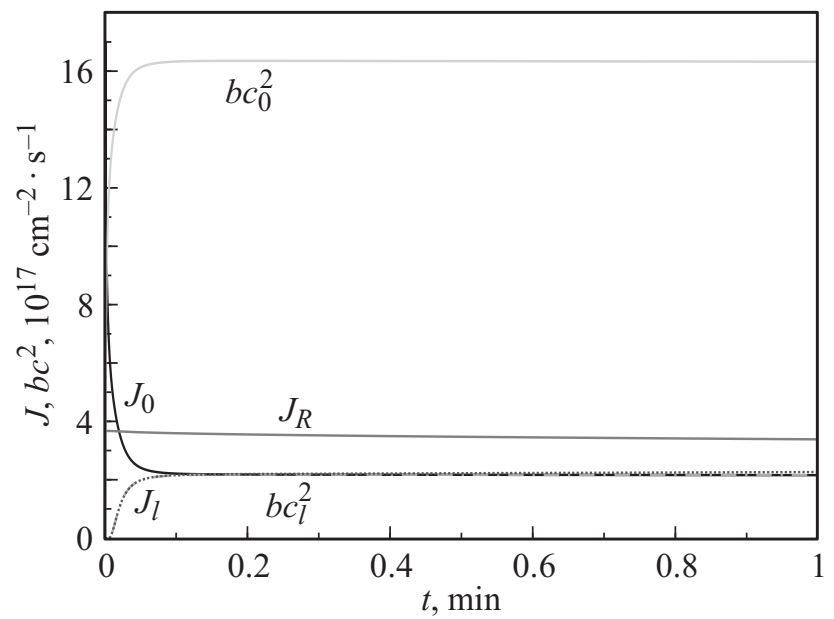

Рис. 6. Динамика потоков, $1 \mathrm{~min} . T=400^{\circ} \mathrm{C}, D=5.5 \cdot 10^{-5}$, $b=2.1 \cdot 10^{-22}, \quad s=5.7 \cdot 10^{-5}, \quad \bar{p}_{0}=34.1, \quad \mu=9.54 \cdot 10^{20}$, $a=0.1, \quad z_{\max }=0.1 \bar{c}=9.4 \cdot 10^{18}, \quad \Gamma=\sqrt{\mu s / b}=1.6 \cdot 10^{19}$, $\Gamma_{\max }=\Gamma(1+0.1)=1.77 \cdot 10^{19}$.

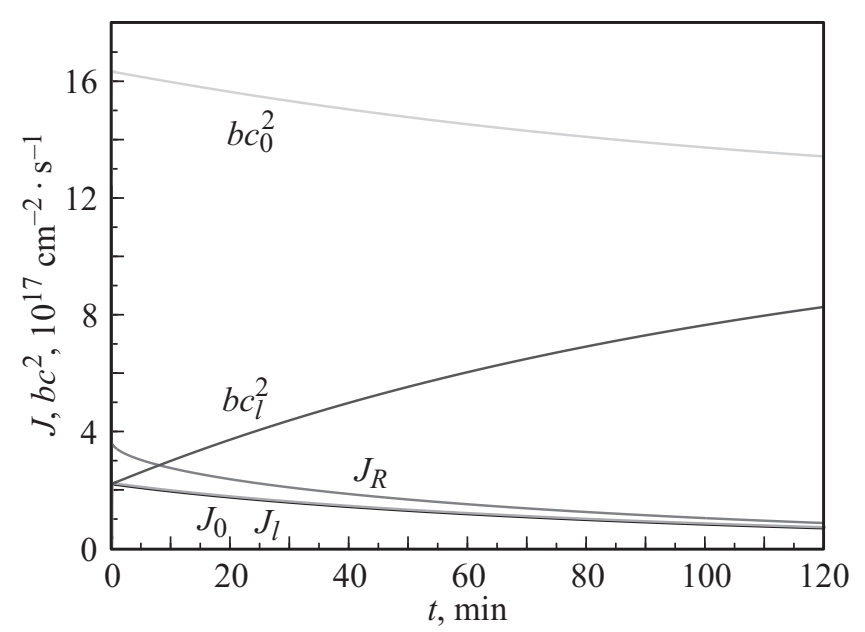

Рис. 7. Динамика потоков, $120 \mathrm{~min} . T=400^{\circ} \mathrm{C}, D=5.5 \cdot 10^{-5}$, $b=2.1 \cdot 10^{-22}, \quad s=5.7 \cdot 10^{-5}, \quad \bar{p}_{0}=34.1, \quad \mu=9.54 \cdot 10^{20}$, $a=0.1, \quad z_{\max }=0.1 \bar{c}=9.4 \cdot 10^{18}, \quad \Gamma=\sqrt{\mu s / b}=1.6 \cdot 10^{19}$, $\Gamma_{\max }=\Gamma(1+0.1)=1.77 \cdot 10^{19}$.

Проанализируем динамику потоков. Вследствие скачкообразного напуска водорода (под достаточно большим давлением) на входе происходит быстрый переходный процесс (рис. 6). Всплеск (плотности) потока $J_{0}(t)=-D \partial_{x} c(t, 0)$ (материал вначале ,пуст ${ }^{66}$ сменяется спадом и стабилизацией. На выходе поток диффузии $J_{l}(t)=-D \partial_{x} c(t, l)$ вначале практически совпадает с десорбционным, поскольку давление в выходном объеме еще пренебрежимо мало для регистрации заметной ресорбции $\mu s p_{l}$. „Слипание“ $J_{0}(t)$, $J_{l}(t)$ говорит о том, что наступил квазистационарный режим: распределение в объеме линейное. Поток водородопроницаемости в приближении Ричардсона $D\left[\bar{c}_{0}(t)-\bar{c}_{l}(t)\right] / l=D \Gamma\left[\sqrt{p_{0}(t)}-\sqrt{p_{l}(t)}\right] / l$ обозначен как $J_{R}$. Локальная стабилизация потоков в масштабе $2 \mathrm{~h}$ (рис. 7) выглядит лишь переходом к длительным монотонным трендам. Потоки $\mu s p_{0, l}$ не отражены в силу баланса $\mu s p_{0, l}-b c_{0, l}^{2}= \pm J_{0, l}$. Заметна ошибка превышения $J_{R}$ над „истинным“ уровнем проницаемости $J_{0}=J_{l}$.

\section{3. Модель быстрой водородопроницаемости}

Приближение Ричардсона в квазистационарном режиме (когда распределение диффундирующего водорода в объеме линейное)

$$
\begin{aligned}
J(t)=-D \partial_{x} c & =D l^{-1}\left[c_{0}(t)-c_{l}(t)\right] \approx J_{R}(t) \\
& =D \Gamma l^{-1}\left[\sqrt{p_{0}(t)}-\sqrt{p_{l}(t)}\right]
\end{aligned}
$$

часто используется при анализе водородопроницаемости (см., в частности, [16,17,20,21]). Выше показано, что в условиях, когда мембрана очень тонкая, а сплав подбирается с целью быстрой проницаемости, подстановка равновесных концентраций приводит к заметной погрешности. Поставим задачу моделирования концентраций $c_{0, l}$ по давлениям $p_{0, l}$ (что представляет и самостоятельный интерес) без квазиравновесного упрощения $c(t)=\Gamma \sqrt{p(t)}$.

За короткое в масштабе эксперимента время $t_{0}$ наступает квазистационарный режим (ловушки насыщены и $\left.\partial_{x} c=-\left[c_{0}(t)-c_{l}(t)\right] / l\right)$ :

$$
\begin{gathered}
\dot{p}_{0, l}(t)=\mp \beta_{0, l}\left[c_{0}(t)-c_{l}(t)\right], \\
\beta_{0, l} \equiv S D\left[\alpha V_{\text {in,out }} l\right]^{-1} T, \\
\mu s p_{0, l}(t)-b c_{0, l}^{2}(t)= \pm D l^{-1}\left[c_{0}(t)-c_{l}(t)\right], \\
t \geq t_{0}>0 .
\end{gathered}
$$

Поскольку $\dot{p}_{l}(t)=-V_{\text {in }} V_{\text {out }}^{-1} \dot{p}_{0}(t) \Rightarrow p_{l}(t)=p_{l}\left(t_{0}\right)+V_{\text {in }} V_{\text {out }}^{-1} \times$ $\times\left[p_{0}\left(t_{0}\right)-p_{0}(t)\right]$, достаточно из граничных условий $(5)$ выразить $c_{0, l}(t)=c_{0, l}\left(p_{0}(t)\right)$ и подставить в первое уравнение (4) (выбор знаков соответствует порядку индексов $0, l)$.

Для численного моделирования удобны безразмерные переменные

$$
\begin{gathered}
X_{0, l}(t)=1+2 l c_{0, l}(t) b D^{-1}, \\
a_{0, l}(t)=4 l^{2} \Gamma^{2} p_{0, l}(t) b^{2} D^{-2}-1 .
\end{gathered}
$$

При этом система уравнений (5) компактно записывается в симметричном виде $a_{0}+2 X_{l}=X_{0}^{2}, a_{l}+2 X_{0}=X_{l}^{2}$. Для переменной $X \equiv X_{l}$ получаем неполное уравнение четвертой степени $\left[X^{2}-a_{l}\right]^{2}=4\left[2 X+a_{0}\right]$, которое решается в радикалах (нас интересует положительный корень). Правда, явное выражение несколько громоздко и все равно придется численно интегрировать первое уравнение (4) вида $\dot{p}_{0}=f\left(p_{0}\right)$. Поэтому нацелимся на вывод дифференциальных уравнений для $X_{0, l}$, поскольку 
информация о динамике граничных концентраций $c_{0, l}$ представляет и самостоятельный интерес.

Продифференцируем по времени уравнения (5) и подставим производные давлений из (4). В переменных $X_{0, l}$ получим систему

$$
\begin{gathered}
\dot{X}_{0}(t)=-s M_{0}\left[X_{0}-X_{l}\right] \cdot \frac{X_{l}-V_{\text {in }} V_{\text {out }}^{-1}}{X_{0} X_{l}-1}, \\
M_{0} \equiv \frac{\mu S T}{\alpha V_{\text {in }}}, \\
\dot{X}_{l}(t)=s M_{l}\left[X_{0}-X_{l}\right] \cdot \frac{X_{0}-V_{\text {in }}^{-1} V_{\text {out }}}{X_{0} X_{l}-1}, \\
M_{l} \equiv \frac{\mu S T}{\alpha V_{\text {out }}} .
\end{gathered}
$$

Сформулируем теперь поэтапно алгоритм численного моделирования при текущих значениях параметров $D$, $b, s$ (авторы пользовались свободно распространяемым пакетом Scilab-5.5.2). Ориентируемся на „обычные“ экспериментальные условия [16-18,20,21], включая параметры $p, T, l, V, S$.

1. Фиксируем $t=t_{0}$ : пропускаем быстрые переходные процессы (в примере выше - десяток секунд в масштабе часов эксперимента). Для переменной $X \equiv X_{l}$ численно решаем неполное уравнение четвертой степени $\left[X^{2}-a_{l}\left(t_{0}\right)\right]^{2}=4\left[2 X+a_{0}\left(t_{0}\right)\right]$. Упомянутый математический пакет, естественно, представит четыре корня, выбираем положительный. Ошибиться трудно, поскольку графически уравнение $X=F(X)$,прозрачно“: ищем пересечение графика элементарной функции с прямой в положительном ортанте. Для уточнений графического приближения (или даже более грубого $X\left[\bar{c}\left(t_{0}\right)\right]$ ) можно воспользоваться простыми итерациями $X_{k+1}=F\left(X_{k}\right)$. Из системы уравнений $a_{0}+2 X_{l}=X_{0}^{2}, a_{l}+2 X_{0}=X_{l}^{2}\left(t=t_{0}\right)$ находим недостающее значение $X_{0}\left(t_{0}\right)$. Формально достаточно и одного уравнения, но учитываем усредняющие процедуры, включая определение значений $p_{0, l}\left(t_{0}\right)$.

2. С полученными начальными данными численно интегрируем систему (7),(8) $\left(t \geq t_{0}\right)$. Это элементарная операция для математических пакетов. Предостережение: данные нужно переопределить как вещественные, иначе начнет накапливаться „мнимая“ составляющая концентрации.

3. Замена переменных (6) определяет концентрации $c_{0, l}(t)$, по которым из уравнений (5) вычисляются модельные давления $p_{0, l}(t)$.

Вычислительные эксперименты показывают, что модельные кривые уже практически неотличимы (при $\left.t \geq t_{0}\right)$ от тех, которые генерируются исходной принятой моделью 1.3 - нелинейной распределенной краевой задачей.

Отметим принципиальное отличие от квазиравновесной модели (приближения Ричардсона), где единственным параметром для аппроксимации экспериментальных давлений является комплекс $D \Gamma$. При реализации описанного выше алгоритма существенными являются все варьируемые параметры исходной модели, влияющие на проницаемость: $D, b, s$. Тем самым упрощенная модель быстрой водородопроницаемости не теряет информацию о рассматриваемых параметрах переноса.

\section{Заключение}

Модель водородопроницаемости ориентирована на задачу выбора материалов для мембранной технологии выделения особо чистого водорода. Физико-технический характер задачи предполагает оценку основных интегральных показателей переноса с учетом ограниченной информативности эксперимента. В частности, параметр максимальной емкости стока $z_{\max }$ не детализирует многообразие ловушек. Мембраны в установках газоразделения относительно тонкие, материал достаточно однороден, с высокой водородопроницаемостью, так что захват носит характер малой поправки.

Итерационное решение нелинейной распределенной краевой задачи для аппроксимации экспериментальных данных (с целью оценки параметров переноса) требует специализированного программного обеспечения. С другой стороны, для тонких мембран с высокой водородопроницаемостью приближение Ричардсона (когда граничные концентрации растворенного водорода считаются квазиравновесными, соответствующими закону Сивертса) позволяет лишь оценить порядок коэффициента проницаемости.

В работе предложена модель быстрой водородопроницаемости в форме системы двух обыкновенных дифференциальных уравнений, которая (за вычетом начального переходного процесса) эквивалентна исходной краевой задаче. Это позволяет быстро и в широком диапазоне „сканировать“ материал: после параметрической идентификации на основе ограниченной экспериментальной информации можно варьировать условия эксплуатации материала вплоть до экстремальных. Разумеется, речь о математическом моделировании с целью оценок параметров переноса, планирования и повышения эффективности необходимых экспериментальных исследований.

Работа выполнена при поддержке РФФИ (грант 15-0100744).

\section{Список литературы}

[1] Водород в металлах / Под ред. Г. Алефельда, В. Фёлькля. М.: Мир, 1981. Т. 1.506 с.; Т. 2. 430 с.

[2] Взаимодействие водорода с металлами /Под ред. А.П. Захарова. М.: Наука, 1987. 296 с.

[3] Писарев А.А., Цветков И.В., Маренков Е.Д., Ярко С.С. Проницаемость водорода через металлы. М.: МИФИ, 2008. $144 \mathrm{c}$.

[4] Черданцев Ю.П., Чернов И.П., Тюрин Ю.И. Методы исследования систем металл-водород. Томск: ТПУ, 2008. $286 \mathrm{c}$. 
[5] Изотопы водорода. Фундаментальные и прикладные исследования / Под ред. А.А. Юхимчука. Саров: РФЯЦВНИИЭФ, 2009. 697 c.

[6] Gabis I.E. // Techn. Phys. 1999. Vol. 44. P. 90-94.

[7] The hydrogen economy / Eds. M. Ball, M. Wietschel. Cambridge University Press, 2009. 646 p.

[8] Varin R.A., Czujko T., Wronski Z.S. Nanomaterials for solid state hydrogen storage. Springer, 2009. 338 p.

[9] Handbook of hydrogen storage: new materials for future energy storage / Edt. M. Hirscher. Wiley-VCH, 2010. 353 p.

[10] Indeitsev D.A., Semenov B.N. // Acta Mechanica. 2008. Vol. 195. P. 295-304.

[11] Evard E., Gabis I., Yartys V.A. // Int. J. Hydrogen Energy. 2010. Vol. 35. P. 9060-9069.

[12] Lototskyy M.V., Yartys V.A., Pollet B.G., Bowman R.C. Jr. // Int. J. Hydrogen Energy. 2014. Vol. 39. P. 5818-5851.

[13] Zaika Yu.V., Bormatova E.P. // Int. J. Hydrogen Energy. 2011. Vol. 36. P. 1295-1305.

[14] Zaika Yu.V., Rodchenkova N.I. // Appl. Math. Modelling. 2009. Vol. 33. P. 3776-3791.

[15] Zaika Yu.V., Rodchenkova N.I. // Math. Modelling. 2012. P. 269-302.

[16] Kojakhmetov S., Sidorov N., Piven V., Sipatov I., Gabis I., Arinov B. // J. Alloys and Compounds. 2015. P. S36-S40.

[17] Dolan M.D. // J. Membrane Science. 2010. Vol. 362. P. 12-28.

[18] Song G., Dolan M.D., Kellam M.E., Liang D., Zambelli S. // J. Alloys and Compounds. 2015. P. 9322-9328.

[19] Даркен Л.С., Гурри Р.В. Физическая химия металлов. М.: Металлургиздат, 1960. $585 \mathrm{c.}$

[20] Terrani K.A., Balooch M., Wongsawaeng D., Jaiyen S., Olander D.R. // J. Nuclear Materials. 2010. Vol. 397. P. 61-68.

[21] Zhang Y., Maeda R., Komaki M., Nishimura C. // J. Membrane Science. 2006. Vol. 269. P. 60-65. 